Artigo Original

Original Article

Nilceneide Ramos Cavalcanti ${ }^{1}$

Bárbara Oliveira Souza ${ }^{1}$

Ana Cristina Côrtes Gama ${ }^{1}$

Adriane Mesquita de Medeiros ${ }^{1}$

Descritores

Voz

Disfonia

Fonoterapia

Motivação

Docentes

Keywords

Voice

Dysphonia

Speech Therapy

Motivation

Faculty

Endereço para correspondência:

Adriane Medeiros de Mesquita

Av. Professor Alfredo Balena, 190,

Sala 249, Santa Efigênia,

Belo Horizonte (MG), Brasil,

CEP: 30130-100.

E-mail: adrianemmedeiros@hotmail.com

Recebido em: Outubro 02, 2017

Aceito em: Janeiro 09, 2018

\section{Efeito do programa integral de reabilitação vocal em professoras com disfonia comportamental}

\author{
Effect of the comprehensive voice \\ rehabilitation program in teachers with \\ behavioral dysphonia
}

\begin{abstract}
RESUMO
Objetivo: analisar os efeitos do Programa Integral de Reabilitação Vocal (PIRV) e os estágios motivacionais durante a terapia de voz em professoras com disfonia comportamental. Método: estudo observacional longitudinal retrospectivo com dados de prontuários de 33 professoras referente à análise perceptivo-auditiva, acústica, autopercepção vocal e Protocolo de Índice de Desvantagem Vocal (IDV-10) nas situações pré e pós-fonoterapia e estágios motivacionais de adesão ao tratamento. Resultados: Na avaliação perceptivo-auditiva da voz, 64,7\% das participantes melhoraram a qualidade vocal pós-fonoterapia. Quando comparados os momentos pré e pós-fonoterapia, observou-se que $82,4 \%$ das professoras melhoraram a voz segundo a autopercepção. O IDV-10 não mostrou diferença entre os momentos pré e pós-fonoterapia ( $\mathrm{p}=0,879)$. Na análise acústica da voz, observou-se melhora da média de todos os parâmetros avaliados. Houve associação estatisticamente significante na análise entre os momentos pré e pós-fonoterapia apenas nas variáveis tempo máximo de fonação e shimmer. A maioria das professoras encontrou-se no estágio de contemplação na escala URICA-VOZ. Não houve significância estatística na análise de associação entre o IDV-10, análise perceptivo-auditiva, tempo máximo de fonação e parâmetros da análise acústica com a autopercepção vocal das professoras e com a avaliação perceptivo-auditiva da voz. Conclusão: o uso do PIRV para tratamento de disfonias comportamentais promoveu resultados positivos quando comparada a avaliação vocal antes e após tratamento. Quanto à motivação, as professoras acreditavam com firmeza na possibilidade de enfrentar o problema, mas ainda sem muito esforço para mudar essa situação.
\end{abstract}

\begin{abstract}
Purpose: Analyze the effects of the Comprehensive Vocal Rehabilitation Program (CVRP) and the motivational stages during speech therapy in teachers with behavioral dysphonia. Methods: Retrospective, longitudinal, observational study using data from the medical records of 33 teachers regarding auditory-perceptual, acoustic, self-perception analyses and the Voice Handicap Index (VHI-10) protocol in pre- and post-speech therapy situations, and motivational stages of adherence to treatment. Results: Auditory-perceptual assessment of the voice showed that $64.7 \%$ of the participants improved voice quality post-speech therapy. Comparison between the pre- and post-speech therapy moments showed that $82.4 \%$ of the teachers improved their voice according to self-perception. The VHI-10 showed no statistically significant difference between the pre- and post-speech therapy moments $(\mathrm{p}=0.879)$. Acoustic analysis of the voice showed improvement in the means of all evaluated parameters. Statistically significant correlation was observed in the analysis between the pre- and post-speech therapy moments only for the variables maximum phonation time and shimmer. Most of the teachers were at the stage of contemplation on the URICA-VOICE scale. No statistical significance was observed in the correlation analysis between VHI-10, auditory-perceptual assessment of the voice, maximum phonation time, and parameters of acoustic analysis with vocal self-perception of teachers and with auditory-perceptual assessment of voice. Conclusion: The use of CVRP for the treatment of behavioral dysphonia presented positive results in the pre-and post-speech therapy evaluation. As for motivation, the teachers strongly believed in the possibility of facing the problem, but still without much effort to change this situation.
\end{abstract}

Trabalho realizado no Departamento de Fonoaudiologia, Faculdade de Medicina, Universidade Federal de Minas Gerais - UFMG - Belo Horizonte (MG), Brasil.

${ }^{1}$ Universidade Federal de Minas Gerais - UFMG - Belo Horizonte (MG), Brasil.

Fonte de financiamento: nada a declarar.

Conflito de interesses: nada a declarar. 


\section{INTRODUÇÃO}

O despreparo vocal para suprir as demandas necessárias do trabalhador é o problema básico da voz profissional falada e é a causa mais comum da disfonia comportamental caracterizada pelo uso excessivo e incorreto da voz. Os professores, profissionais da voz falada, apresentam alterações cumulativas que limitam seu desempenho profissional no decorrer de sua profissão e podem levá-los a apresentar disfonia funcional ou organofuncional, também chamada de comportamental ${ }^{(1)}$.

A disfonia é definida como uma alteração da comunicação oral determinada pela dificuldade de emissão vocal que restringe a produção natural da voz ${ }^{(1)}$. Diante da demanda, das condições de trabalho e da falta de preparo vocal o professor utiliza muitas vezes ajustes vocais inadequados como, por exemplo, variações na frequência; manutenção da intensidade aumentada para competir com o ruído da sala de aula e chamar a atenção dos alunos, gerando falhas na voz, rouquidão, esforço para falar, secura na garganta e pigarro ${ }^{(2-4)}$.

Diante deste contexto, o trabalho da fonoaudiologia com os professores considera a voz como um instrumento de trabalho e, por isso, necessita de cuidados para que não sofra interferência de fatores desencadeadores de alterações. É importante também abordar a voz como expressão e, como tal, o trabalho envolve o potencial dos professores, garantindo que eles percebam a riqueza dos efeitos que podem ser gerados por meio da voz na interlocução com as pessoas, sempre adequando os parâmetros vocais de acordo com as demandas sociais, pessoais e profissionais do indivíduo ${ }^{(5)}$. Pode-se dizer então que o trabalho da fonoaudiologia com professores, tem como finalidade conscientizar e empoderar o profissional quanto ao desenvolvimento do potencial comunicativo e vocal, além de observar os fatores ambientais e organizacional do trabalho docente.

O protocolo de avaliação clínica das disfonias consta com avaliação perceptivo-auditiva, videoestroboscopia, análise acústica, obtenção das medidas aerodinâmicas e percepção do paciente sobre seu problema de voz. Tal padronização permite que os resultados das avaliações sejam passíveis de comparação com os descritos na literatura, independentemente do tipo de tratamento empregado ${ }^{(6)}$. Além disso, outros protocolos como o IDV-10 ${ }^{(7)}$ e o URICA-VOZ ${ }^{(8,9)}$ têm sido utilizados para auxiliar na compreensão da desvantagem da voz em diversos domínios da vida e da motivação do indivíduo sobre a sua capacidade de mudança comportamental relacionada à saúde.

A terapia fonoaudiológica ou fonoterapia é considerada como a melhor forma de tratamento para as disfonias comportamentais e tem como objetivo orientar o docente quanto às noções básicas de higiene vocal e cuidados com a voz, abuso e mau uso da voz e indicar estratégias para favorecer um ambiente de trabalho melhor, além de adequar a resistência vocal. Para esse processo terapêutico pode-se utilizar programas contendo sessões de terapias definidas, como por exemplo, o Programa Integral de Reabilitação Vocal - PIRV ${ }^{(10)}$.

O presente estudo tem por objetivo analisar os efeitos do Programa Integral de Reabilitação Vocal e os estágios motivacionais durante a terapia de voz em professoras com disfonia comportamental.

\section{MÉTODO}

Trata-se de um estudo observacional longitudinal retrospectivo de professores da rede pública de ensino com disfonia comportamental. O estudo foi realizado com prontuários de professoras atendidas no Projeto de extensão universitária realizado no Ambulatório de Fonoaudiologia da Universidade Federal de Minas Gerais (UFMG). Todos os indivíduos envolvidos assinaram o Termo de Consentimento Livre e Esclarecido (TCLE).

Os critérios de inclusão para o estudo foram: professores da rede municipal de ensino, do gênero feminino, com idade entre 18 e 55 anos, com queixa de disfonia, sem lesão laríngea ao exame de videolaringoscopia e que foram encaminhados pelo setor de saúde ocupacional. Os critérios de exclusão considerados foram: professores com outro tipo de distúrbio de fala ou linguagem, distúrbios hormonais, disfonias psicogênicas, psiquiátricas ou espasmódicas, profissionais da voz cantada e profissionais com histórico de diagnóstico de problemas neurológicos ou quadro agudo laríngeo recente.

As professoras com disfonia funcional foram atendidas durante oito semanas com sessões de 40 minutos de duração. As sessões desenvolvidas seguem o PIRV e trabalham aspectos vocais como corpo-voz, fonte glótica, ressonância, coordenação pneumofonoarticulatória e atitude comunicativa em sessões com orientações e abordagens alternativas incentivando a prática diária dos exercícios propostos e proporcionando a aprendizagem motora ${ }^{(10)}$.

Para a coleta de dados foram utilizadas as seguintes informações contidas nos prontuários: análise perceptivo-auditiva, análise acústica, autopercepção vocal no momento inicial e final do programa, análise da autopercepção de desvantagem vocal e dos estágios motivacionais ao tratamento dos professores.

As avaliações perceptivo-auditiva e acústica da voz foram feitas antes e após a fonoterapia para mensurar o resultado da intervenção fonoterápica. Além dessas avaliações, foi utilizado o Protocolo de Índice de Desvantagem Vocal: 10 (IDV-10) ${ }^{(7)}$ aplicado pré e pós fonoterapia para identificar a desvantagem vocal do professor e a Escala URICA-VOZ ${ }^{(9)}$ aplicada na quarta sessão, para identificação de estágios de motivação para a mudança vocal. A autopercepção do professor foi verificada por meio da questão "Circule como você avalia sua voz", sendo que as opções de resposta foram: excelente, muito boa, boa, razoável e ruim.

Para as avaliações perceptivo-auditivas das vozes foram coletadas amostras da vogal sustentada /a/. As avaliações foram realizadas por duas fonoaudiólogas com mais de 15 anos de experiência na área de voz. A forma de avaliação foi por consenso e por tarefa de comparação. Para cada par de vozes os juízes foram instruídos a responder se elas eram iguais ou qual delas melhorou ou piorou, e ainda, informar o grau geral da disfonia $(\mathrm{G})$ das duas vozes, utilizando-se o parâmetro perceptivo-auditivo da escala GRBAS, graduado em uma escala Likert de quatro pontos: $0=$ ausente; $1=$ leve; $2=$ moderado; e $3=$ intenso $^{(11)}$. Os avaliadores não tiveram conhecimento prévio se a voz analisada era pré ou pós-exercício ou a qual momento da terapia pertencia. 
Para a análise das medidas acústicas foram coletadas emissão de vogal sustentada/a/ com frequência e intensidade habituais. Foi realizada a eliminação do início e do fim da emissão, em decorrência das irregularidades contidas nestes trechos.

Os registros vocais foram gravados diretamente em um computador da marca Dell ${ }^{\circledR}$, modelo Optiplex GX260, equipado com placa de som profissional Direct Sound ${ }^{\circledR}$, com a utilização de um microfone do tipo condensador da marca Shure ${ }^{\circledR} 16 \mathrm{~A}$, posicionado a $10 \mathrm{~cm}$ do canto da boca do paciente, em posição diagonal, com ângulo de captação direcional de $45^{\circ}$ por meio do programa Audacity ${ }^{\circledR}$, em cabina acústica.

Os parâmetros acústicos selecionados foram tempo máximo de fonação (TMF), frequência fundamental média (F0), Jitter (\%), quociente de perturbação da frequência (PPQ \%), Shimmer (\%), quociente de perturbação da amplitude (APQ \%) e proporção harmônico-ruído $(\mathrm{NHR} \mathrm{dB})^{(12)}$. O software utilizado para a análise acústica foi o CSL, com o programa Multi-Dimensional Voice Program (MDVP) da Kay Pentax ${ }^{\circledR}$.

Segundo o programa MDVP da Kay Pentax ${ }^{\circledR}$ os parâmetros acústicos selecionados podem ser definidos a partir dos seguintes aspectos, tais como: o valor da frequência fundamental média, que é a média de todos os períodos da frequência extraídos; o jitter expresso em porcentagem, que é o valor da média relativa da variação da frequência em relação ao período; o PPQ expresso em porcentagem, que consiste na média relativa da perturbação da frequência de cinco em cinco períodos (média de cinco pontos); shimmer expresso em porcentagem, que é a média relativa da variabilidade de amplitude, pico a pico; o APQ em porcentagem, que é a média relativa da variabilidade da amplitude de 11 a 11 períodos (média de 11 pontos) e o NHR que relaciona o componente harmônico com o componente de ruído da onda acústica. Os valores de normalidade segundo o programa encontram-se no quadro 1.

O Protocolo IDV-10 é composto por 10 questões, produz um escore total único calculado por meio de somatória simples das respostas de suas questões, podendo variar de zero a 40 pontos, sendo zero o indicativo de nenhuma desvantagem e 40 de desvantagem máxima. Cada questão deve ser respondida em uma escala de cinco pontos, sendo zero nunca e quatro sempre ${ }^{(7)}$.

A Escala URICA ${ }^{(8)}$ foi adaptada para a área de voz em 2013, sendo chamada de URICA-VOZ. A escala é composta por 32 itens com quatro grupos (pré-contemplação, contemplação, ação e manutenção) de 8 afirmações. A escala tem como objetivo verificar em que estágio motivacional de enfrentamento da reabilitação vocal encontra-se um paciente com disfonia. Os estágios motivacionais avaliados pela escala são: pré-contemplação
(PC) - o indivíduo ainda não percebe que há um problema a ser enfrentado; contemplação (C) - o sujeito acredita firmemente na viabilidade de enfrentar o problema, mas não se esforça para mudar essa situação; ação (A) - o indivíduo apresenta as tentativas perceptíveis para a mudança e manutenção $(\mathrm{M})$ - não há retrocesso com relação à terapia, sendo constante o esforço contínuo por parte do indivíduo para preservar a mudança alcançada. O cálculo da escala URICA consiste em uma média simples das marcações das afirmações de cada grupo, excluindo-se do cálculo as questões 4, 9, 20, 1 e 31. Portanto, para cada grupo realiza-se média de sete questões.

Após o cálculo das médias de cada estágio, aplica-se a seguinte fórmula: (Média de C + Média de A + Média de M) - Média de PC. A classificação nos estágios depende da pontuação de corte que são: $\leq 8$ - para os sujeitos em fase de pré-contemplação; de 8 a 11 - para os sujeitos em fase de contemplação e de 11 a 14 - para os sujeitos que estão dispostos a uma mudança em relação ao problema ${ }^{(9)}$.

A amostra de conveniência foi composta por todas as 33 professoras atendidas no período de agosto de 2014 a dezembro de 2015.

Para a compilação dos resultados foi realizada a análise descritiva dos dados e medidas de associação entre as variáveis utilizando os testes Qui-Quadrado de Pearson ou Exato de Fisher e T de Student com nível de significância de $5 \%(\mathrm{p} \leq 0,05)$. Todas as análises foram realizadas no Software IBM-SPSS Statistic Base, versão 19.

Este trabalho foi analisado pelo Comitê de Ética em Pesquisa (COEP) da Universidade Federal de Minas Gerais (UFMG) e aprovado com o número CAAE44359215.5.0000.5149.

\section{RESULTADOS}

Todos participantes da pesquisa eram do gênero feminino $(n=33)$ e a média de idade foi de 39,2 anos $(\mathrm{DP}=7,68)$. Em relação ao tempo de docência, cinco $(15,2 \%)$ professoras participantes do estudo possuíam menos de cinco anos, 15 (45,5\%) de seis a 15 anos e, $13(39,4 \%)$ de 16 a 31 anos.

$\mathrm{Na}$ avaliação perceptivo-auditiva da voz realizada por consenso entre duas fonoaudiólogas, foi possível observar que $21(63,6 \%)$ participantes melhoraram, seis $(18,2 \%)$ não mudaram e seis $(18,2 \%)$ apresentaram piora da qualidade vocal pós-fonoterapia. A avaliação fonoaudiológica verificou melhora na voz das professoras comparando os momentos pré e pós-fonoterapia ( $\mathrm{p}=0,019)$.

Quadro 1. Valores de normalidade dos parâmetros acústicos segundo o programa MDVP da Kay Pentax ${ }^{\circledR}$

\begin{tabular}{|c|c|c|}
\hline Parâmetro & Normalidade Voz Feminina & Normalidade Voz Masculina \\
\hline $\mathbf{F}_{\mathbf{0}}$ & $243,973 \mathrm{~Hz}$ & $145,223 \mathrm{~Hz}$ \\
\hline PPQ & $0,366 \%$ & $0,338 \%$ \\
\hline Jitter & $0,633 \%$ & $0,589 \%$ \\
\hline Shimmer & $1,997 \%$ & $2,523 \%$ \\
\hline APQ & $1,397 \%$ & $1,986 \%$ \\
\hline $\mathbf{N H}$ & 0,112 & 0,122 \\
\hline
\end{tabular}

Legenda: $\mathrm{F}_{0}=$ Frequência Fundamental Média; $\mathrm{PPQ}=$ Quociente de Perturbação da Frequência; $\mathrm{APQ}=$ quociente de perturbação da amplitude; NHR dB = proporção harmônico-ruído 
Na tabela 1 os valores referentes à autopercepção vocal dos professores pré e pós-fonoterapia foram descritos para 31 participantes do estudo. Observou-se que a maioria apontou melhora da autopercepção vocal $(87,1 \%)$. Também houve redução no número de professores que relatou percepção vocal como razoável/ruim no início da terapia. As professoras perceberam melhora na voz comparando o relato nos momentos pré e pós-fonoterapia $(\mathrm{p}=0,008)$.

Em relação aos resultados do Protocolo IDV-10 a média total obtida pelos participantes pré e pós-fonoterapia foi de $6,45(\mathrm{DP}=4,86)$ e 6,39 $(\mathrm{DP}=6,47)$, respectivamente. Quando comparado os dois momentos, observou-se que 19 pacientes $(57,6 \%)$ melhoraram os seus índices de desvantagem vocal e os demais $(42,4 \%)$ mantiveram valores similares. Entretanto, a associação entre os momentos pré e pós-fonoterapia não foi estatisticamente significante $(\mathrm{p}=0,951)$.

A tabela 2 mostra os valores referentes às médias e ao desvio padrão do tempo máximo de fonação, frequência fundamental, APQ, NHR, PPQ, Shimmer e Jitter dos participantes. Quando comparado os momentos pré e pós-fonoterapia, observou-se que a média dos participantes indicou melhora do tempo máximo de fonação (72,7\%), APQ (66,7\%), NHR (60,6\%), PPQ (63,6\%), Shimmer (63,6\%) e Jitter (66,7\%). Entretanto, houve associação estatisticamente significante na análise entre os momentos pré e pós-fonoterapia apenas nas variáveis tempo máximo de fonação, frequência fundamental e Shimmer.

Foi possível observar que a média do escore da escala URICA-Voz foi de 8,35 (DP=3,82) para o grupo de professoras avaliadas. Em relação aos estágios da escala URICA-VOZ (Tabela 3), a maioria das professoras encontrou-se no estágio de contemplação $(54,83 \%)$. Nenhum dos participantes do estudo estava no estágio de manutenção.

Não houve significância estatística na análise de associação entre as variáveis utilizadas no estudo (IDV, análise perceptivo-auditiva da voz, tempo máximo de fonação e parâmetros da análise acústica da voz) com a autopercepção vocal dos professores (Tabela 4) e com a avaliação perceptivo-auditiva da voz realizadas pelas fonoaudiólogas (Tabela 5).

Tabela 1. Distribuição de frequência da autopercepção vocal dos professores

\begin{tabular}{lccc}
\hline & Pré-Fonoterapia & Pós-Fonoterapia & Valor P \\
\hline Excelente/Muito Boa/Boa & $20(64,5 \%)$ & $27(87,1 \%)$ & $0,008^{*}$ \\
Razoável/Ruim & $11(35,5 \%)$ & $4(12,9 \%)$ & \multirow{2}{*}{} \\
\hline
\end{tabular}

Teste Qui-Quadrado (IC = 95\%); *Valores Significativos $(\mathrm{p} \leq 0,05)$

Tabela 2. Análise de associação entre as médias das medidas acústicas nos momentos pré e pós-fonoterapia

\begin{tabular}{|c|c|c|c|c|c|}
\hline & Pré-fonoterapia & Desvio Padrão & Pós-fonoterapia & Desvio Padrão & Valor $\mathrm{p}$ \\
\hline Tempo Máximo de Fonação & 9,23 & 3,00 & 11,65 & 3,77 & $0,001^{*}$ \\
\hline Frequência Fundamental & 190,24 & 28,22 & 201,07 & 29,17 & $0,027^{*}$ \\
\hline $\mathrm{APQ}$ & 6,99 & 14,62 & 3,80 & 1,36 & 0,230 \\
\hline NHR & 0,15 & 0,48 & 0,14 & 0,23 & 0,190 \\
\hline$P P Q$ & 0,66 & 0,54 & 0,55 & 0,34 & 0,304 \\
\hline Shimmer & 6,20 & 2,55 & 5,26 & 2,18 & $0,026^{*}$ \\
\hline Jitter & 3,75 & 15,15 & 0,97 & 0,62 & 0,303 \\
\hline
\end{tabular}

Teste T de Student; *Valores Significativos $(p \leq 0,05)$

Legenda: $P P Q=$ Quociente de Perturbação da Frequência; $A P Q=$ Quociente de Perturbação da Amplitude; NHR dB = proporção harmônico-ruído

Tabela 3. Autopercepção dos estágios motivacionais no tratamento de voz

\begin{tabular}{lcc}
\multicolumn{1}{c}{ URICA-Voz (Estágios Motivacionais) } & $\mathrm{n}$ & \% \\
\hline Pré-Contemplação & 11 & 35,49 \\
Contemplação & 17 & 54,83 \\
Ação & 3 & 9,68 \\
Manutenção & 0 & 31 \\
Total & 0 & 100 \\
\hline
\end{tabular}

O valor de $n=31$ refere-se a dados faltantes de duas professoras

Tabela 4. Distribuição de frequência e associação entre as variáveis explicativas (protocolo IDV, análise perceptivo-auditiva da voz e parâmetros da análise acústica da voz) e a autopercepção vocal

\begin{tabular}{|c|c|c|c|c|}
\hline \multirow{3}{*}{ Variáveis } & \multirow{3}{*}{ Total de Participantes } & \multicolumn{2}{|c|}{ Autopercepção vocal } & \multirow{3}{*}{ Valor $\mathrm{p}$} \\
\hline & & Melhor & Igual ou Pior & \\
\hline & & $\mathrm{n}(\%)$ & $\mathrm{n}(\%)$ & \\
\hline \multicolumn{5}{|l|}{ IDV } \\
\hline Melhor pós-fonoterapia & 21 & $8(38,1 \%)$ & $13(61,9 \%)$ & 0,337 \\
\hline Igual ou pior pós-fonoterapia & 10 & $4(40,0 \%)$ & $6(60,0 \%)$ & \\
\hline
\end{tabular}

Teste Qui-Quadrado ou Exato de Fisher (IC = 95\%); As diferenças no total de participantes referem-se a dados faltantes nos prontuários dos professores Legenda: IDV = Índice de Desvantagem Vocal; PPQ = Quociente de Perturbação da Frequência; $A P Q=Q$ Quociente de Perturbação da Amplitude; NHR dB = proporção harmônico-ruído 
Tabela 4. Continuação...

\begin{tabular}{|c|c|c|c|c|}
\hline \multirow{3}{*}{ Variáveis } & \multirow{3}{*}{ Total de Participantes } & \multicolumn{2}{|c|}{ Autopercepção vocal } & \multirow{3}{*}{ Valor $p$} \\
\hline & & Melhor & Igual ou Pior & \\
\hline & & $\mathrm{n}(\%)$ & $\mathrm{n}(\%)$ & \\
\hline \multicolumn{5}{|c|}{ Análise Perceptivo-Auditiva da Voz } \\
\hline Melhor pós-fonoterapia & 19 & $8(42,1 \%)$ & $11(57,9 \%)$ & 0,906 \\
\hline Igual ou pior pós-fonoterapia & 12 & $4(33,3 \%)$ & $8(66,7 \%)$ & \\
\hline \multicolumn{5}{|l|}{ Tempo Máximo de Fonação } \\
\hline \multicolumn{5}{|l|}{ APQ } \\
\hline Melhor pós-fonoterapia & 21 & $8(38,1 \%)$ & $13(61,9 \%)$ & 0,943 \\
\hline Igual ou pior pós-fonoterapia & 10 & $5(50,0 \%)$ & $6(60,0 \%)$ & \\
\hline \multicolumn{5}{|l|}{ NHR } \\
\hline Melhor pós-fonoterapia & 19 & $6(31,6 \%)$ & $13(68,4 \%)$ & 0,623 \\
\hline Igual ou pior pós-fonoterapia & 11 & $5(45,5 \%)$ & $6(54,5 \%)$ & \\
\hline \multicolumn{5}{|l|}{ Shimmer } \\
\hline Melhor pós-fonoterapia & 20 & $8(40,0 \%)$ & $12(60,0 \%)$ & 0,816 \\
\hline Igual ou pior pós-fonoterapia & 11 & $5(45,5 \%)$ & $6(54,5 \%)$ & \\
\hline \multicolumn{5}{|l|}{ Jitter } \\
\hline Melhor pós-fonoterapia & 21 & $7(33,3 \%)$ & $14(66,7 \%)$ & 0,588 \\
\hline Igual ou pior pós-fonoterapia & 9 & $5(55,6 \%)$ & $4(44,4 \%)$ & \\
\hline
\end{tabular}

Teste Qui-Quadrado ou Exato de Fisher (IC = 95\%); As diferenças no total de participantes referem-se a dados faltantes nos prontuários dos professores Legenda: IDV = Índice de Desvantagem Vocal; PPQ = Quociente de Perturbação da Frequência; $A P Q=Q$ Quociente de Perturbação da Amplitude; NHR dB = proporção harmônico-ruído

Tabela 5. Distribuição de frequência e associação entre as variáveis explicativas (protocolo IDV, tempo máximo de fonação e parâmetros da análise acústica da voz) e a análise perceptivo-auditiva da voz

\begin{tabular}{|c|c|c|c|c|}
\hline \multirow{3}{*}{ Variáveis } & \multirow{3}{*}{ Total de Participantes } & \multicolumn{2}{|c|}{ Avaliação perceptivo-auditiva } & \multirow{3}{*}{ Valor $p$} \\
\hline & & Voz melhor & Voz igual ou pior & \\
\hline & & $\mathrm{n}(\%)$ & $\mathrm{n}(\%)$ & \\
\hline \multicolumn{5}{|l|}{ IDV } \\
\hline Melhor pós-fonoterapia & 23 & $15(65,2 \%)$ & $8(34,8 \%)$ & 0,906 \\
\hline Igual ou pior pós-fonoterapia & 9 & $6(66,6 \%)$ & $4(44,4 \%)$ & \\
\hline \multicolumn{5}{|l|}{ Tempo Máximo de Fonação } \\
\hline \multicolumn{5}{|l|}{ APQ } \\
\hline Melhor pós-fonoterapia & 22 & $13(59,1 \%)$ & $9(40,9 \%)$ & 0,196 \\
\hline Igual ou pior pós-fonoterapia & 10 & $8(60,0 \%)$ & $2(20,0 \%)$ & \\
\hline \multicolumn{5}{|l|}{ NHR } \\
\hline Melhor pós-fonoterapia & 20 & $14(70 \%)$ & $6(30 \%)$ & 0,473 \\
\hline Igual ou pior pós-fonoterapia & 11 & $6(54,5 \%)$ & $5(45,5 \%)$ & \\
\hline \multicolumn{5}{|l|}{ Shimmer } \\
\hline Melhor pós-fonoterapia & 21 & $14(66,7 \%)$ & $7(33,3 \%)$ & 0,304 \\
\hline Igual ou pior pós-fonoterapia & 11 & $7(63,6 \%)$ & $4(36,4 \%)$ & \\
\hline \multicolumn{5}{|l|}{ Jitter } \\
\hline Melhor pós-fonoterapia & 22 & $15(68,2 \%)$ & $7(31,8 \%)$ & 0,916 \\
\hline Igual ou pior pós-fonoterapia & 9 & $5(55,6 \%)$ & $4(44,4 \%)$ & \\
\hline
\end{tabular}

Teste Qui-Quadrado ou Exato de Fisher (IC = 95\%); As diferenças no total de participantes referem-se a dados faltantes nos prontuários dos professores

Legenda: $P P Q=$ Quociente de Perturbação da Frequência; $A P Q=$ quociente de perturbação da amplitude; NHR Db = proporção harmônico-ruído 


\section{DISCUSSÃO}

As professoras participantes da pesquisa apresentaram melhora nos aspectos clínicos e na autopercepção vocal após a realização do PIRV. Há forte evidência cientifica da eficácia na reabilitação vocal por meio desse programa para o tratamento de disfonias comportamentais quando comparado a outro método ${ }^{(13)}$. Ressalta-se o resultado do estudo com praticamente os mesmos participantes da pesquisa que mostrou indicativos de boa adesão e satisfação com o PIRV, apesar da dificuldade na realização dos exercícios em casa, conforme a recomendação ${ }^{(14)}$.

$\mathrm{Na}$ avaliação perceptivo-auditiva foi possível observar que a maioria $(63,6 \%)$ das professoras apresentou melhora na qualidade vocal. O fato de as professoras deste estudo apresentarem disfonia funcional reforça o benefício dessa abordagem para esse grupo ocupacional com quadro clínico específico, prevenindo o aparecimento de lesões nas pregas vocais. Estudo realizado com dois grupos de profissionais da voz de diversas ocupações comparou a efetividade do PIRV com o Método de Exercícios de Função Vocal (ESV), e encontrou melhora na qualidade vocal em ambas as intervenções. O PIRV foi apontado como o que obteve um melhor resultado no final do programa em relação ao ESV, mas não houve diferença significativa ${ }^{(13)}$. Outro estudo comparou a avaliação vocal, laríngea e autorpecepção, de 42 professores com disfonia funcional ou organofuncional no momento pré e pós-fonoterapia com abordagem eclética e constatou melhora da voz em $73,8 \%$ dos $\operatorname{casos}^{(15)}$.

Ao analisar a percepção das professoras referente à própria voz nos momentos pré e pós-fonoterapia contatou-se que as participantes em sua maioria $(87,1 \%)$ referiram melhora na voz após o tratamento, corroborando outros estudos ${ }^{(15,16)}$. Ressalta-se que o relato de melhora da voz identificada pelas professoras estava relacionado principalmente à redução da rouquidão e fadiga vocal. A proposta do PIRV considera que ao identificar o problema, conscientizar e modificar os hábitos vocais negativos, com propostas de técnicas de associação corpo-voz, ajuste glótico e equilíbrio ressonantal, o tratamento de voz pode ser efetivo ${ }^{(10)}$.

Quando comparados os momentos pré e pós-fonoterapia observou-se que mais de $50 \%$ dos pacientes melhoraram os seus índices de desvantagem vocal, confirmando os resultados encontrados em pesquisas que utilizaram o mesmo protocolo de autopercepção vocal ${ }^{(13,16)}$. Deve-se considerar que as professoras mesmo no momento pré-fonoterapia apresentaram pouca desvantagem vocal, o que pode ter interferido na ausência de diferença entre os momentos pré e pós-fonoterapia. As professoras atendidas foram encaminhadas ao Ambulatório de Fonoaudiologia pelo setor de saúde ocupacional do município, com o quadro clínico de disfonia em estágio inicial e, algumas professoras, sem queixa vocal, não se configurando uma demanda espontânea de tratamento, o que precisa ser considerado na análise dos resultados.

O uso de protocolos de autopercepção vocal ajudam o paciente a mensurar a dimensão do seu problema ${ }^{(17)}$. A eficácia dessa avaliação se confirma quando ao final do tratamento o paciente relata a evolução de sua qualidade vocal diminuindo o impacto da desvantagem decorrente da alteração vocal.
Com relação à análise acústica as professoras obtiveram melhora em todas as medidas acústicas analisadas quando comparado os momentos pré e pós-fonoterapia. As medidas de TMF, Shimmer e frequência fundamental apresentaram diferença estatisticamente significante. Estudos atuais apontam alteração nas medidas acústicas dos professores no momento inicial da fonoterapia, sem relato das mudanças ocorridas após a reabilitação ${ }^{(18,19)}$. Há uma escassez de estudos quanto aos resultados das medidas acústicas pós-fonoterapia em professores. Estudo com 42 pacientes com disfonia comportamental encontrou melhora do jitter, quociente de perturbação de frequência, shimmer e quociente de perturbação de amplitude após fonoterapia. Apenas $\mathrm{a}_{0}$ e NHR não se diferenciou entre os dois momentos ${ }^{(15)}$.

Neste estudo, houve aumento dos valores de $\mathrm{F}_{0}$ no pós-fonoterapia que se mantiveram dentro da faixa da normalidade. Este resultado é frequentemente encontrado em casos de disfonias comportamentais com vozes moderadamente alteradas ${ }^{(17)}$. Os valores da $\mathrm{F}_{0}$ reduzidos podem ser observados em pacientes disfônicos cuja etiologia esteja relacionada a um comportamento vocal inadequado ${ }^{(20,21)}$.

Outro parâmetro de avaliação analisado neste estudo foi o TMF - que pode ser usado como um meio de diagnóstico, acompanhamento e evolução de terapia em pacientes disfônicos ${ }^{(12)}$. Verificou-se que após o programa houve aumento do TMF das professoras desta pesquisa. Outro estudo com 17 professoras municipais com disfonia verificou melhora do TMF após a realização do programa EFV de Stemple e Gerdeman ${ }^{(22)}$.

A análise acústica é uma ferramenta importante na avaliação clínica do paciente disfônico ${ }^{(12)}$, portanto, o seu uso isolado para a avaliação da voz pode ser um fator limitante no diagnóstico. Diante disso, não é viável utilizar apenas essa ferramenta para avaliar os efeitos da terapia.

Na aplicação da escala URICA-VOZ a maioria dos participantes concentrou-se no estágio motivacional de contemplação. É importante ressaltar que, neste estudo, a escala foi aplicada na quarta sessão de terapia. Estudos revelam diferenças nos estágios motivacionais entre professores e pacientes disfônicos que ainda não participaram de terapia vocal ou estão em tratamento de voz, sendo que os estágios de pré-contemplação e contemplação são os mais encontrados. O UIRCA-VOZ não foi reaplicado no final do tratamento limitando a possibilidade de se conhecer a possível mudança do estágio motivacional com a intervenção realizada.

Em uma pesquisa em que a escala URICA-VOZ foi aplicada em 138 professoras com queixas vocais da rede de educação municipal de Montes Claros/MG o resultado apontou que a maioria $(59,4 \%)$ das participantes se encontravam no estágio de pré-contemplação ${ }^{(23)}$. Vale ressaltar, que essas professoras ainda não tinham se submetido a qualquer terapia de voz. Outra pesquisa com 151 pacientes disfônicos que se submeteram à triagem vocal no ambulatório de voz de uma instituição pública avaliou o estágio de prontidão para mudança em que os participantes se encontravam no momento da triagem e foi observado que a maioria $(76,2 \%)$ se concentrou no estágio de contemplação ${ }^{(24)}$. 
Desta maneira, confirmando o achado deste estudo uma pesquisa feita com 66 pacientes que estavam iniciando tratamento fonoaudiológico há, pelo menos, uma semana após avaliação inicial, em ambulatórios de voz de Hospitais Universitários de duas instituições de ensino em Fonoaudiologia, apontou que $57,6 \%$ dos participantes encontravam-se no estágio de contemplação $0^{(9)}$. Pode-se inferir que o tempo em que os pacientes estão em terapia influencia no estágio de motivação, já que aqueles que se concentram no primeiro estágio (pré-contemplação) são indivíduos que ainda não tem consciência de que há um problema a ser enfrentado.

Nossa experiência na aplicação de um programa de reabilitação vocal mostrou que, nos casos de professoras com disfonia funcional, o PIRV supre as demandas vocais e ao ser apresentado com sessões definidas, promove maior adesão à terapia, conforme outro estudo realizado com a mesma população ${ }^{(14)}$. Diante disto, é necessário realizar novas pesquisas envolvendo o programa como forma de prevenção de lesões secundárias de pregas vocais.

A efetividade de programas de reabilitação vocal para professores precisa ser mais explorada comparando grupos com quadros clínicos distintos, como disfonia funcional e organofuncional. Existem poucos estudos longitudinais que avaliam a evolução da terapia identificando os aspectos quantitativos e qualitativos do programa utilizado, possíveis de serem conhecidos no presente estudo. Destaca-se que a pesquisa apontou melhora em todos os aspectos vocais trabalhados em professoras com disfonia comportamental sem lesão secundária de prega vocal, mas os resultados não podem ser generalizados. O tamanho da amostra reduzido pode ter afetado as associações encontradas com redução do poder estatístico do teste. Apesar de o protocolo URICA-VOZ não ter sido aplicado em dois momentos do Programa dificultando a análise da mudança do estágio motivacional dos professores participantes, verificou-se uma melhor prontidão desses para enfrentar o problema de voz.

\section{CONCLUSÃO}

O uso do PIRV para tratamento de disfonias comportamentais promoveu resultados positivos quando comparada à avaliação vocal antes e após tratamento. Na análise dos estágios motivacionais, os professores concentraram-se no estágio motivacional de contemplação, ou seja, acreditavam com firmeza na possibilidade de enfrentar o problema, mas ainda sem muito esforço para mudar essa situação.

\section{REFERÊNCIAS}

1. Behlau M, Azevedo R, Pontes P. Conceito de voz normal e classificação das disfonias. In: Behlau M. Voz: o livro do especialista, v. 1. Rio de Janeiro: Revinter; 2005. p. 53-84.

2. Ricarte $A$, Bommarito $S$, Chiari B. Impacto vocal de professores. Rev CEFAC. 2011;13(4):719-27. http://dx.doi.org/10.1590/S1516-18462011005000014.

3. Ferreira LP, Arruda AF, Marquezin DMSS. Expressividade oral de professoras: análise de recursos vocais. Distúrb Comun. 2012;24(2):223-37.
4. Azevedo LL, Martins PC, Mortimer EF, Quadros AL, Sá EF, Moro L, et al. Recursos de expressividade usados por uma professora universitária. Distúrb Comun. 2014;26(4):777-89.

5. Ferreira LP, Dragone MLS, Simões-Zenari M. Giannini A. Teoria e a Prática promotoras da Voz do Professor. In: Oliveira IB, Almeida AAF, Raize T, Behlau M. Atuação fonoaudiológica em voz profissional. São Paulo: Roca; 2011. p. 1-20.

6. Dejonckere PH, Bradley P, Clemente P, Cornut G, Crevier-Buchman L, Friedrich $\mathrm{G}$, et al. A basic protocol for functional assessment of voice pathology, especially for investigating the efficacy of (phonosurgical) treatments and evaluating new assessment techniques: guideline elaborated by the Committee on Phoniatrics of the European Laryngological Society (ELS). Eur Arch Otorhinolaryngol. 2001;258(2):77-82. http://dx.doi. org/10.1007/s004050000299. PMid:11307610.

7. Costa T, Oliveira G, Behlau M. Validation of the Voice Handicap Index: 10 (VHI-10) to the Brazilian Portuguese. Codas. 2013;25(5):482-5. http:// dx.doi.org/10.1590/S2317-17822013000500013. PMid:24408554.

8. Teixeira LC, Rodrigues AL, Silva AF, Azevedo R, Gama ACC, Behlau $M$. The use of the URICA-VOICE questionnaire to identify the stages of adherence to voice treatment. CoDAS. 2013;25(1):8-15. PMid:24408164.

9. Costa CVC. Estágios motivacionais e sua correlação com sintomas de ansiedade e pacientes com disfonia [dissertação]. São Paulo: Universidade de São Paulo, Faculdade de Odontologia de Bauru; 2015. http://dx.doi org/10.11606/D.25.2015.tde-03062015-084513.

10. Behlau M, Pontes P, Vieira VP, Yamasaki R, Madazio G. Apresentação do Programa Integral de Reabilitação Vocal para o tratamento das disfonias comportamentais. CoDAS. 2013;25(5):492-6. http://dx.doi.org/10.1590/ S2317-17822013000500015. PMid:24408556.

11. Hirano M. Clinical examination of voice. New York: Springer Verlag; 1981. p. 81-4.

12. Behlau M, Azevedo R, Pontes P. Avaliação de voz. In: Behlau M. Voz: o livro do especialista. Rio de Janeiro: Revinter; 2010. p. 139-147.

13. Pedrosa V, Pontes A, Pontes P, Behlau M, Peccin SM. The Effectiveness of the comprehensive voice rehabilitation program compared with the vocal function exercises method in behavioral dysphonia: a randomized clinical trial. J Voice. 2016;30(3):377.e11-9. http://dx.doi.org/10.1016/j. jvoice.2015.03.013. PMid:25959424.

14. Souza BO, Tonon IG, Souza EVS, Nogueira BDFM, Silva SP, Ribeiro $\mathrm{K}$, et al. Adesão e satisfação de professores participantes do Programa Integral de Reabilitação Vocal. Distúrb Comun. 2017;29(2):284-91. http:// dx.doi.org/10.23925/2176-2724.2017v29i2p284-291 .

15. Ribeiro MB, Gama ACC, Bassi IB, Teixeira LC. Parâmetros vocais, laríngeos e de autopercepção de professoras disfônicas: análise após tratamento fonoaudiológico. Rev CEFAC. 2013;15(3):631-41. http://dx.doi org/10.1590/S1516-18462013000300016.

16. Lemos IO, Marchand DLP, Cassol M. Índice de Desvantagem Vocal pré e pós-intervenção vocal em pacientes disfônicos. Audiol Commun Res. 2015;20(4):355-60. http://dx.doi.org/10.1590/2317-6431-2015-1580.

17. Behlau M, Madazio G, Moreti F, Oliveira G, Santos LM, Paulinelli BR, et al. Efficiency and cutoff values of self-assessment instruments on the impact of a voice problem. J Voice. 2015;1997(15):121-6. https:// www.ncbi.nlm.nih.gov/entrez/query.fcgi? $\mathrm{cmd}=$ Retrieve \&db=PubMed\&1 ist_uids $=26168902 \&$ dopt $=$ Abstract

18. Ribeiro VV, Cielo CA. Medidas vocais perceptivo-auditivas e acústicas, queixas vocais e características profissionais de professoras de Santa Maria (RS). Audiol Commun Res. 2014;19(4):387-98. http://dx.doi.org/10.1590/ S2317-64312014000400001395.

19. Pereira ERBN, Tavares ELM, Martins RHG. Voice Disorders in teachers: clinical, videolaryngoscopical, and vocal aspects. J Voice. 2015;29(5):564 71. http://dx.doi.org/10.1016/j.jvoice.2014.09.019. PMid:25704475.

20. Rubin JS, Blake E, Mathieson L. Musculoskeletal patterns in patients with voice disorders. J Voice. 2007;21(4):477-84. http://dx.doi.org/10.1016/j. jvoice.2005.02.001. PMid:16626934. 
21. Angsuwarangsee T, Morrison M. Extrinsic laryngeal muscular tension in patients with voice disorders. J Voice. 2002;16(3):333-43. http://dx.doi. org/10.1016/S0892-1997(02)00105-4. PMid:12395986.

22. Mendonça RA, Sampaio TMM, Provenzano L. Medida do tempo máximo de fonação de professoras do município de Niterói/RJ. Ver CEFAC. 2012;14(6):1204-8. http://dx.doi.org/10.1590/S151618462012005000103

23. Rossi-Barbosa LA, Gama ACC, Caldeira AP. Associação entre prontidão para mudanças de comportamento e queixa de disfonia em professores. CoDAS. 2015;27(2):170-7. http://dx.doi.org/10.1590/2317-1782/20152013088. PMid:26107083.
24. Lopes LW, Vilela EG. Autoavaliação e prontidão para mudança em pacientes disfônicos. CoDAS. 2016;28(3):295-301. http://dx.doi.org/10.1590/2317$1782 / 20162015111$.

\section{Contribuições dos autores}

$N R C$ realizou a coleta dos dados, a redação do manuscrito e a aprovação da versão final; BOS auxiliou na análise dos dados, na redação do manuscrito e na aprovação da versão final; ACCG participou da orientação do trabalho, redação do manuscrito e aprovação da versão final; AMM participou da concepção do estudo, análise dos dados, orientou todas as etapas do trabalho e redação do manuscrito e aprovação da versão final. 